\title{
Advanced aircraft manufacturing and maintenance using three-dimensional printing
}

\author{
Tin-Chih Toly Chen ${ }^{1} \cdot$ Seung-Kyum Choi $^{2}$. Seung Ki Moon ${ }^{3} \cdot$ Yu-Cheng Wang ${ }^{4}$ \\ Published online: 11 November 2019 \\ (C) Springer-Verlag London Ltd., part of Springer Nature 2019
}

Three-dimensional (3D) printing is a process that uses computer-aided design (CAD) data to achieve the continuous layered deposition of different shapes to produce 3D objects [1]. Consequently, 3D-printed objects can have any geometric shapes and features. 3D printing has been applied in numerous industries in which the traditional manufacturing processes are relatively complex, have long cycle times, and are difficult to maintain precision [2]. The applications of $3 \mathrm{D}$ printing technologies have overcome these problems to reduce the manufacturing costs and shorten cycle times [2].

This special issue is focused on the application of 3D printing to the aircraft industry. The applications of 3D printing in the aircraft industry differ from those in other industries in the following aspects: oligopoly, projectbased production, long cycle times, and the pursuit of lighter products. Nevertheless, to date, 3D printing, as a green production technology, has benefitted the aircraft industry with shortened cycle times, reduced production

Tin-Chih Toly Chen

tolychen@ms37.hinet.net

Seung-Kyum Choi

schoi@me.gatech.edu

Seung Ki Moon

skmoon@ntu.edu.sg

Yu-Cheng Wang

tony.cobra@msa.hinet.net

1 Department of Industrial Engineering and Management, National Chiao Tung University, Hsinchu, Taiwan

2 Woodruff School of Mechanical Engineering, Georgia Institute of Technology, Atlanta, USA

3 School of Mechanical and Aerospace Engineering, Nanyang Technological University, Singapore, Singapore

4 Department of Aeronautical Engineering, Chaoyang University of Science and Technology, Taichung, Taiwan costs, and lighter component weights. In particular, the lightweight of airplanes reduces the fuel consumption and in turn elevates profitability. In addition, the aviation market demands that spare parts be delivered rapidly. By 3D printing spare parts domestically, the need for transporting spare parts to meet the local demand is eliminated, thereby the cycle time can be dramatically shortened. However, the following concerns must still be addressed:

(1) The aircraft industry is a high-tech industry. Many countries or regions require but lack the capability to manufacture, maintain, or repair aircraft parts on their own; 3D printing provides an opportunity for these countries or regions to gain this capability at low cost in a short time. To such countries or regions, an introduction of the current practice of applying 3D printing to the aircraft industry is required.

(2) The application of 3D printing in the aircraft industry is ongoing. Some efforts should be made to clarify which related topics are more critical, feasible, or useful [3].

(3) Rapid advances in 3D printing technologies have been observed in the recent years. As a result, the previous reviews on similar topics may become out-of-date [4].

This special issue is an attempt to address these concerns. It is intended to provide technical details of applying advanced $3 \mathrm{D}$ printing technologies to aircraft manufacturing and maintenance, including the current practices, challenges faced, and opportunities that can enhance the effectiveness of the existing applications. These details will hold great interest for researchers in advanced manufacturing, aviation engineering, mechanical engineering, $\mathrm{CAD} / \mathrm{CAM} / \mathrm{CAE}$, sustainability, technology management, and for practicing managers and engineers. This special issue features a balance between state-of-the-art research and practical applications. This special issue also provides a forum for researchers and 
practitioners to review and disseminate quality research work on advanced 3D printing applications to aircraft manufacturing and maintenance and the critical issues for further development. According to strict review criteria, ten articles from researchers around the world were accepted.

In the first paper, Liu, Weng, Bi, Chew, Liu, Ma, and Moon investigated how to deposit functionally graded material (FGM) onto a cast iron substrate using laseraided additive manufacturing (LAAM). They deposited Inconel 625 nickel-base superalloy on the substrate as a buffer layer, on which the stainless steel 420 (SS420) layers were subsequently grown. In this way, the cracking issue was successfully addressed.

Stereolithography (SLA)-based 3D printing has good performances in surface finish and precision, and therefore plays an important role in the aircraft industry. In the second paper, He, Landowne, Currie, Amoah, Shi, Yunus, and Liu proposed a 3D printing technology that enabled a UV projector to continuously cure resin while scanning over the build area. According to the experimental results, the proposed SLA technology had advantages over existing large-scale digital light processing (DLP) technologies in terms of the printing speed and material flexural properties.

In the third paper, Wang, Chen, and Yeh reviewed the current $3 \mathrm{D}$ printing practices in the aircraft industry and identified five factors critical to the applicability of advanced 3D printing technologies to the aircraft industry. Subsequently, they proposed a fuzzy systematic approach to compare the relative importance of the identified factors and assess the applicability of an advanced 3D printing technology to the aircraft industry.

To make weak rigid large thin-walled aerospace parts, mirror milling systems have been considered as a green and efficient alternative to traditional processing methods. In the fourth paper, Xiao, Zhao, Guo, Huang, and Lin designed a novel dual-robot mirror milling system consisting of a machining hybrid robot, a supporting hybrid robot, and fixtures. They also proposed a collaborative machining approach by establishing a relative pose relationship between the cutter and the supporting head. In this way, the cutter trajectory of the machining robot could be generated in real time according to the end trajectory.

3D printing applications can enhance not only the competitiveness but also the sustainability of an aircraft manufacturing or maintenance, repair, and overhaul (MRO) company. In the fifth paper, Lin and Chen discussed the long-term opportunities and challenges facing an aircraft manufacturing or MRO company planning to apply 3D printing technologies, so as to identify the critical factors for enhancing the sustainability of a
3D printing application to an aircraft manufacturing or MRO company.

In the sixth paper, Chen and Wang proposed an advanced fuzzy approach for forecasting the yield of a 3D-printed aircraft part. The advanced fuzzy approach derived the direct solution (DS) versions of existing fuzzy yield learning models by dealing with the original yield value directly, thereby optimizing the forecasting performance.

Fabricating parts using contour parallel toolpaths with variable bead widths has been shown to be a viable way to increase the production speed and improve the geometry accuracy. In the seventh paper, to manufacture void-free parts, Xiong, Park, Padmanathan, Dharmawan, Foong, Rosen, and Soh established a comprehensive process planning framework for determining adaptive contour parallel toolpaths with variable bead widths. In the framework, the level-set method was used as the toolpath planning algorithm, and a Gaussian process regression model was applied to process planning for generating the desired bead geometry.

The design freedom afforded by $3 \mathrm{D}$ printing (or additive manufacturing) is ideal for the space industry, where parts are made in small volume and often highly customized. Sacco and Moon reviewed the literature and current practice of additive manufacturing for space (AMFS). The main materials adopted for space-based AMFS were polymers, while those for ground-based AMFS included metal, polymer, and others (regolith, cement, and ceramic).

In a selective laser melting (SLM) process, the building of a process window is a fundamental task for fabricating a full-dense part with a new material. To fulfill this task, a single-track test (STT) has been widely applied due to its simplicity. However, the stable process parameters obtained from STTs cannot ensure a successful fabrication in practice. To address this issue, in the ninth paper, Ahn proposed a new multi-layered fabrication approach to build a process window.

Directed energy deposition (DED) is an additive manufacturing technology suitable for freeform production with metallic materials. However, it is a challenging task to achieve a satisfactory powder distribution. To address this issue, in the last paper, Takemura, Koike, Kakinuma, Sato, and Oda analyzed the gas flow under a powder nozzle by applying a computational fluid dynamics (CFD) simulation.

I would like to thank the IJAMT Editor-in-Chief, Andrew Y. C. Nee, for fully supporting the release of this special issue. I am also grateful to the contributors who shared their research as well as to the reviewers who spared their valuable time to review papers. I would also like to thank the journal's staff. Without their support and professional assistance, prepublication would not have been possible. 


\section{References}

1. Vaezi M, Seitz H, Yang S (2013) A review on 3D micro-additive manufacturing technologies. Int J Adv Manuf Technol 67(5-8): $1721-1754$

2. Wu HC, Chen TCT (2018) Quality control issues in 3D-printing manufacturing: a review. Rapid Prototyp J 24(3):607-614

3. Chen TCT, Lin YC (2019) A three-dimensional-printing-based agile and ubiquitous additive manufacturing system. Robot Comput Integr Manuf 55:88-95
4. Chen T, Lin YC (2017) Feasibility evaluation and optimization of a smart manufacturing system based on 3D printing: a review. Int $\mathrm{J}$ Intell Syst 32(4):394-413

Publisher's note Springer Nature remains neutral with regard to jurisdictional claims in published maps and institutional affiliations. 